\title{
Stemoninines from the Roots of Stemona tuberosa
}

Li-Gen Lin, ${ }^{\dagger}$ Qiong-Xing Zhong, ${ }^{\ddagger}$ Tin-Yan Cheng, ${ }^{\S}$ Chun-Ping Tang, ${ }^{\dagger}$ Chang-Qiang $\mathrm{Ke},{ }^{\dagger} \mathrm{Ge}$ Lin, ${ }^{\S}$ and Yang $\mathrm{Ye}^{*}{ }^{\dagger}$

${ }^{\dagger}$ State Key Laboratory of Drug Research, Shanghai Institute of Materia Medica, Chinese Academy of Sciences, 555 Zu-Chong-Zhi Road, Zhangjiang Hi-Tech Park, Shanghai 201203, P. R. China, and ${ }^{\ddagger}$ Department of Biology, Hainan Normal University, 99 South Long-Kun Road, Haikou 571158, P. R. China, and ${ }^{\S}$ Department of Pharmacology, The Chinese University of Hong Kong, Hong Kong SAR, P. R. China

\section{Supporting Information}

$1 \square$ Page 2 Figure 5 X-ray diffraction of compound 1

$2 \square$ Page 3-12 X-ray diffraction data tables for bisdehydrostemoninine (1)

$3 \square$ Page 13-22 X-ray diffraction data tables for bisdehydrostemoninine A 
Figure $5 \mathrm{X}$-ray diffraction of compound 1

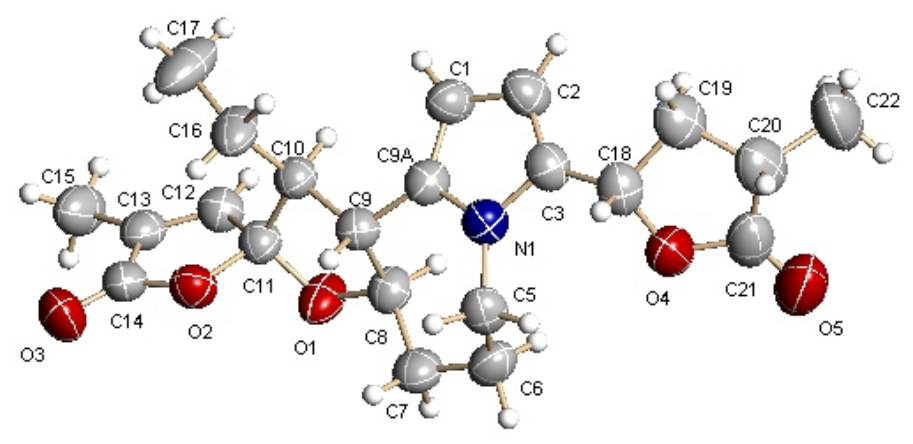

Crystallographic data for the structure reported have been deposited with Cambridge Crystallographic Data Centre (deposition number CCDC 603427). Copies of the data can be obtained, free of charge, on application to the Director, CCDC, 12 Union Road, Cambridge CB2 1EZ, UK (fax: $+44-(0) 1223-336033$ or e-mail: deposite@ccdc.cam.ac.uk). 


\section{X-ray diffraction data tables for bisdehydrostemoninine (1)}

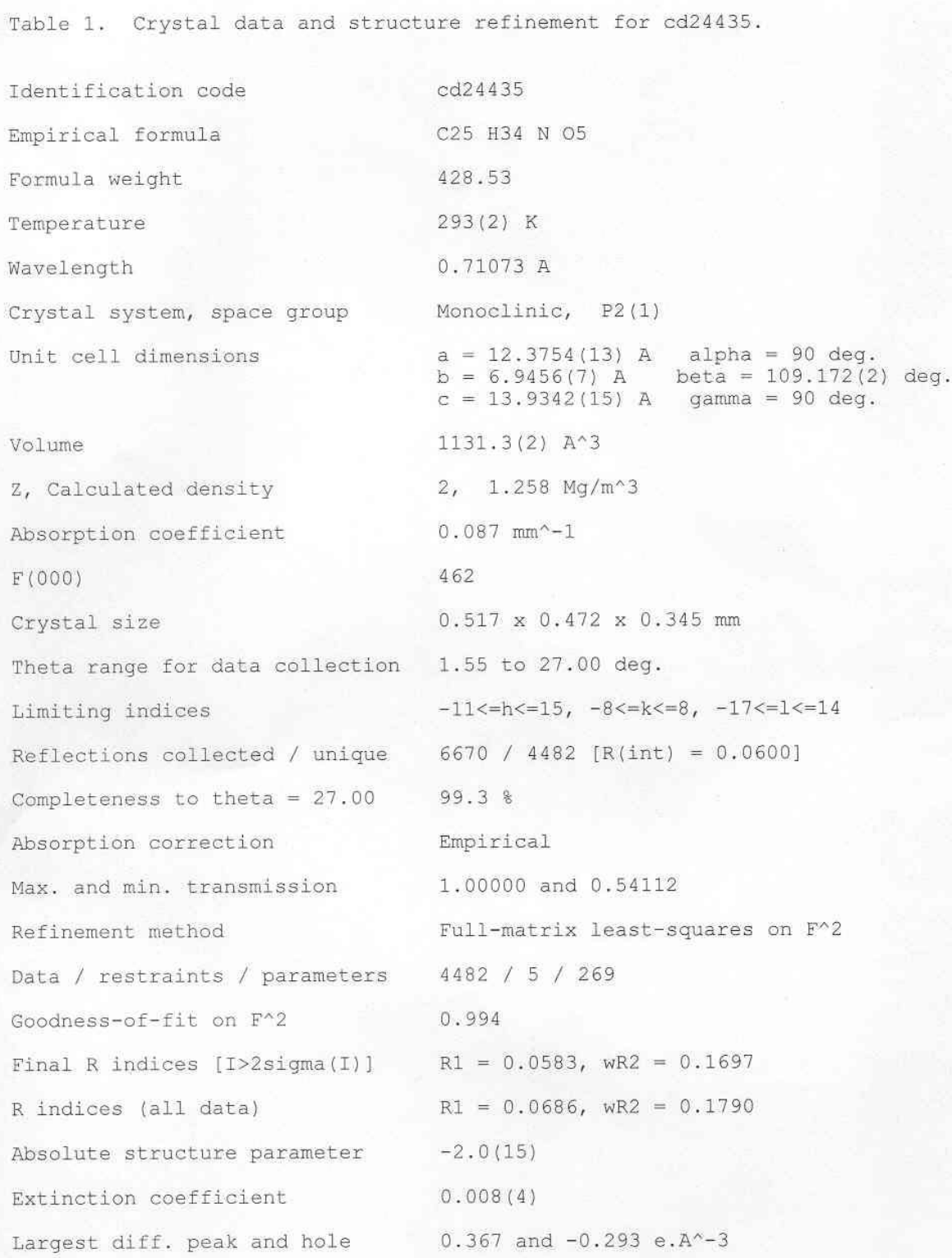


Table 2. Atomic coordinates $\left(x 10^{\wedge} 4\right)$ and equivalent isotropic displacement parameters $\left(A^{\wedge} 2 \times 10^{\wedge} 3\right)$ for cd24435.

$\mathrm{U}(\mathrm{eq})$ is defined as one third of the trace of the orthogonalized Uij tensor.

\begin{tabular}{|c|c|c|c|c|}
\hline & $x$ & y & $z$ & $\mathrm{U}(\mathrm{eq})$ \\
\hline $\begin{array}{l}O(1) \\
O(2) \\
O(3) \\
O(4) \\
O(5) \\
N(1) \\
C(1) \\
C(2) \\
C(3) \\
C(5) \\
C(6) \\
C(7) \\
C(8) \\
C(9) \\
C(10) \\
C(11) \\
C(12) \\
C(13) \\
C(14) \\
C(15) \\
C(16) \\
C(17) \\
C(18) \\
C(19) \\
C C(20) \\
C(21) \\
C(22) \\
C(23) \\
C(24) \\
C(25) \\
C(9 A)\end{array}$ & $\begin{array}{r}-912(2) \\
-2368(2) \\
-4149(2) \\
4549(2) \\
6278(3) \\
1967(2) \\
1030(2) \\
2182(2) \\
2755(2) \\
2208(3) \\
2086(3) \\
886(2) \\
207(2) \\
-46(2) \\
-1171(2) \\
-1764(2) \\
-2682(2) \\
-3670(2) \\
-3487(2) \\
-4817(3) \\
-1822(3) \\
-2794(3) \\
3963(2) \\
4684(3) \\
5873(3) \\
5647(3) \\
6669(4) \\
9615(13) \\
10132(11) \\
9345(11) \\
929(2)\end{array}$ & $\begin{array}{l}8006(3) \\
6702(3) \\
7367(4) \\
4527(4) \\
5579(6) \\
3979(3) \\
2508(5) \\
1923(5) \\
2839(5) \\
5339(5) \\
7428(5) \\
8181(5) \\
7147(4) \\
5038(4) \\
4663(4) \\
6606(4) \\
7053(4) \\
7329(4) \\
7153(4) \\
7735(6) \\
2904(5) \\
2314(6) \\
2661(5) \\
1305(6) \\
2158(7) \\
4234(7) \\
1725(12) \\
2840(20) \\
840(20) \\
-900(20) \\
3792(4)\end{array}$ & $\begin{array}{r}1790(2) \\
473(1) \\
-517(2) \\
3166(2) \\
4035(2) \\
2114(2) \\
3010(2) \\
3375(2) \\
2828(2) \\
1400(2) \\
1654(2) \\
1406(2) \\
1962(2) \\
1658(2) \\
1846(2) \\
1564(2) \\
2013(2) \\
1307(2) \\
314(2) \\
1384(3) \\
1306(3) \\
1683(5) \\
2886(2) \\
3671(3) \\
3932(3) \\
3742(3) \\
4996(3) \\
4790(11) \\
5154(10) \\
4536(9) \\
2237(2)\end{array}$ & $\begin{array}{r}69(1) \\
62(1) \\
90(1) \\
78(1) \\
116(1) \\
57(1) \\
65(1) \\
68(1) \\
61(1) \\
62(1) \\
68(1) \\
66(1) \\
57(1) \\
52(1) \\
54(1) \\
54(1) \\
57(1) \\
59(1) \\
62(1) \\
85(1) \\
75(1) \\
111(2) \\
67(1) \\
82(1) \\
90(1) \\
83(1) \\
128(2) \\
255(5) \\
220(4) \\
231(5) \\
57(1)\end{array}$ \\
\hline
\end{tabular}


Table 3. Bond lengths [A] and angles [deg] for cd24435.

\begin{tabular}{|c|c|}
\hline $\begin{array}{l}O(1)-C(11) \\
O(1)-C(8) \\
O(2)-C(14) \\
O(2)-C(11) \\
O(3)-C(14) \\
O(4)-C(21) \\
O(4)-C(18) \\
O(5)-C(21) \\
N(1)-C(9 A) \\
N(1)-C(3) \\
N(1)-C(5) \\
C(1)-C(9 A) \\
C(1)-C(2) \\
C(1)-H(1) \\
C(2)-C(3) \\
C(2)-H(2) \\
C(3)-C(18) \\
C(5)-C(6) \\
C(5)-H(5 A) \\
C(5)-H(5 B) \\
C(6)-C(7) \\
C(6)-H(6 A) \\
C(6)-H(6 B) \\
C(7)-C(8) \\
C(7)-H(7 A) \\
C(7)-H(7 B) \\
C(8)-C(9) \\
C(8)-H(8) \\
C(9)-C(9 A) \\
C(9)-C(10) \\
C(9)-H(9) \\
C(10)-C(16) \\
C(10)-C(11) \\
C(10)-H(10) \\
C(11)-C(12) \\
C(12)-C(13) \\
C(12)-H(12) \\
C(13)-C(14) \\
C(13)-C(15) \\
C(15)-H(15 A) \\
C(15)-H(15 B) \\
C(15)-H(15 C) \\
C(16)-C(17) \\
C(16)-H(16 A) \\
C(16)-H(16 B) \\
C(17)-H(17 A) \\
C(17)-H(17 B) \\
C(17)-H(17 C) \\
C(18)-C(19) \\
C(18)-H(18) \\
C(19)-C(20) \\
C(19)-H(19 A) \\
C(19)-H(19 B) \\
C(20)-C(21) \\
C(20)-C(22) \\
C(20)-H(20) \\
C(22)-H(22 A) \\
C(22)-H(22 B) \\
C(22)-H(22 C) \\
C(23)-C(24) \\
C(23)-C(25) \# 1 \\
C(24)-C(25) \\
C(25)-C(23) \# 2\end{array}$ & 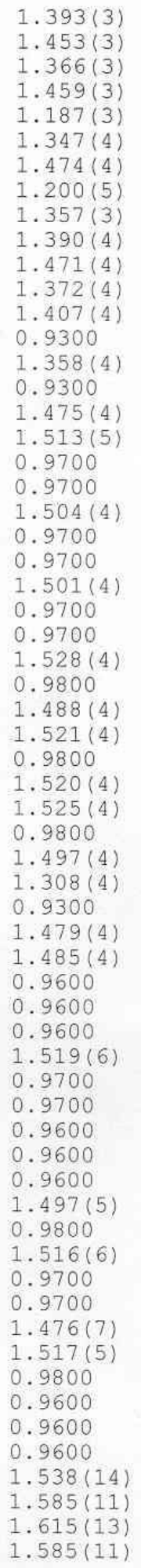 \\
\hline
\end{tabular}




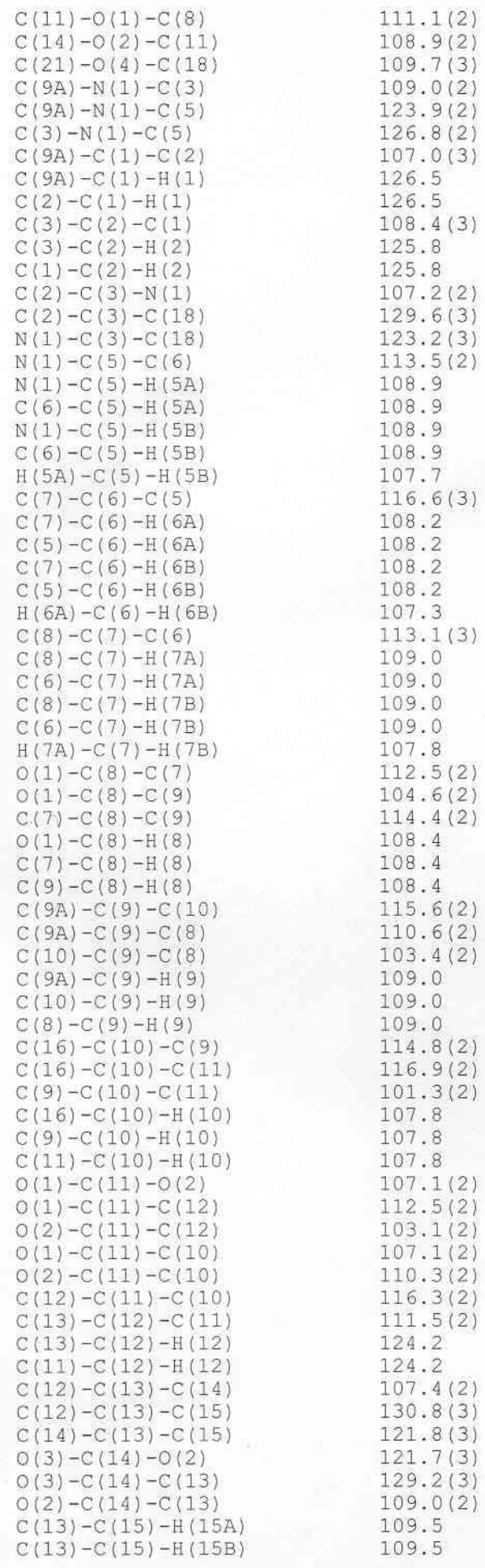




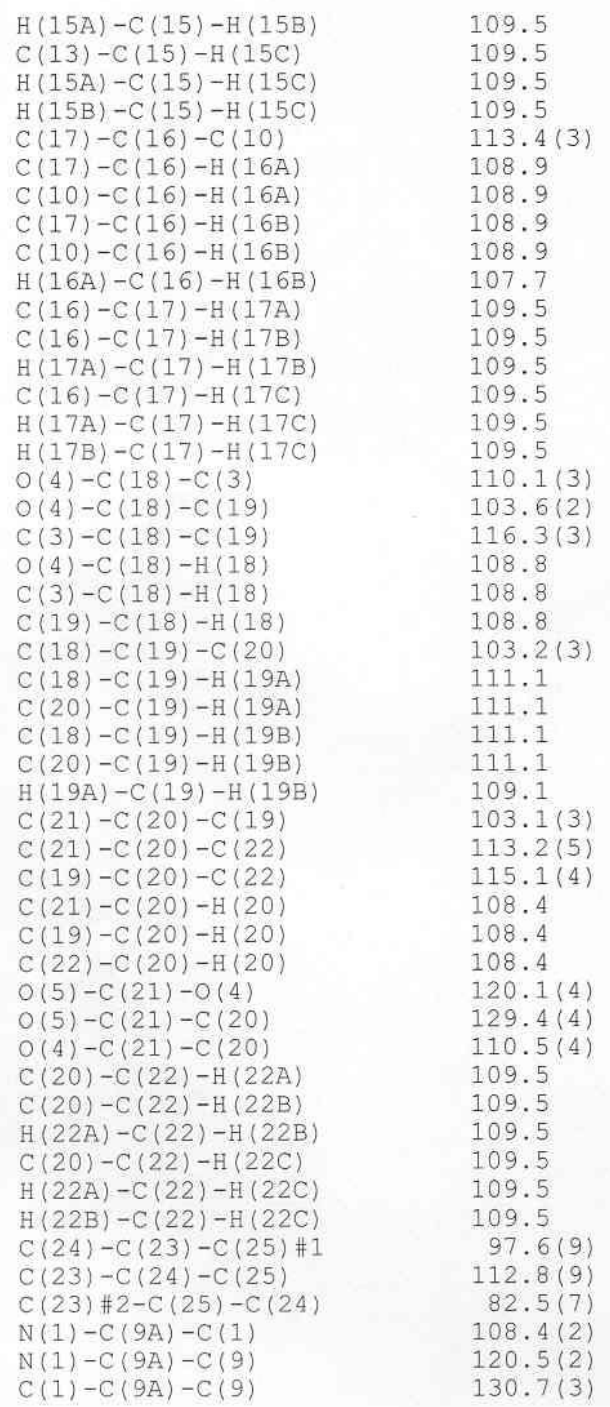

Symmetry transformations used to generate equivalent atoms: $\# 1-x+2, y+1 / 2,-z+1 \quad \# 2-x+2, y-1 / 2,-z+1$ 
Table 4. Anisotropic displacement parameters ( $A^{\wedge} 2 \times 10^{\wedge} 3$ ) for cd24435. The anisotropic displacement factor exponent takes the form:

$-2 \mathrm{pi}^{\wedge} 2\left[\mathrm{~h}^{\wedge} 2 \mathrm{a}^{\star \wedge} 2 \mathrm{U11}+\ldots+2 \mathrm{~h} k \mathrm{a}^{\star} \mathrm{b}^{\star} \mathrm{U} 12\right]$

\begin{tabular}{|c|c|c|c|c|c|c|}
\hline & U11 & $\mathrm{U} 22$ & U33 & $\mathrm{U} 23$ & U13 & U12 \\
\hline$O(1)$ & $58(1)$ & $44(1)$ & $104(2)$ & $-10(1)$ & $24(1)$ & $-1(1)$ \\
\hline$O(2)$ & $64(1)$ & $66(1)$ & $59(1)$ & $7(1)$ & $24(1)$ & $3(1)$ \\
\hline$O(3)$ & $76(1)$ & $115(2)$ & $68(1)$ & $21(1)$ & $7(1)$ & $-1(1)$ \\
\hline$O(4)$ & $65(1)$ & $88(2)$ & $79(1)$ & $9(1)$ & $23(1)$ & $-4(1)$ \\
\hline $0(5)$ & $93(2)$ & $150(3)$ & $96(2)$ & $6(2)$ & $18(2)$ & $-35(2)$ \\
\hline $\mathrm{N}(1)$ & $58(1)$ & $63(1)$ & $56(1)$ & $-2(1)$ & $25(1)$ & $4(1)$ \\
\hline$C(1)$ & $60(2)$ & $66(2)$ & $75(2)$ & $10(1)$ & $30(1)$ & $4(1)$ \\
\hline$C(2)$ & $63(2)$ & $72(2)$ & $68(2)$ & $12(2)$ & $22(1)$ & $14(2)$ \\
\hline$C(3)$ & $58(2)$ & $66(2)$ & $59(1)$ & -1 (1) & $19(1)$ & $7(1)$ \\
\hline$C(5)$ & $59(2)$ & $76(2)$ & $55(1)$ & $2(1)$ & $26(1)$ & $2(1)$ \\
\hline$C(6)$ & $65(2)$ & $74(2)$ & $71(2)$ & $2(1)$ & $29(1)$ & $-14(1)$ \\
\hline$C(7)$ & $70(2)$ & $52(2)$ & $76(2)$ & $2(1)$ & $24(1)$ & $-4(1)$ \\
\hline$C(8)$ & $55(1)$ & $54(1)$ & $62(1)$ & $-8(1)$ & $19(1)$ & $-2(1)$ \\
\hline$C(9)$ & $57(1)$ & $51(1)$ & $51(1)$ & $-3(1)$ & $21(1)$ & $1(1)$ \\
\hline$C(10)$ & $52(1)$ & $49(1)$ & $59(1)$ & $0(1)$ & $18(1)$ & $-2(1)$ \\
\hline$C(11)$ & $53(1)$ & $51(1)$ & $58(1)$ & $-2(1)$ & $16(1)$ & $-1(1)$ \\
\hline$C(12)$ & $61(2)$ & $53(2)$ & $59(1)$ & $-2(1)$ & $22(1)$ & $3(1)$ \\
\hline$C(13)$ & $57(2)$ & $49(1)$ & $73(2)$ & $9(1)$ & $25(1)$ & $0(1)$ \\
\hline$C(14)$ & $61(2)$ & $58(2)$ & $65(2)$ & $10(1)$ & $17(1)$ & $-6(1)$ \\
\hline$C(15)$ & $59(2)$ & 91 (2) & $112(3)$ & $26(2)$ & $36(2)$ & $10(2)$ \\
\hline$C(16)$ & $65(2)$ & $45(2)$ & $104(2)$ & $-5(2)$ & $13(2)$ & $-5(1)$ \\
\hline$C(17)$ & $80(2)$ & $61(2)$ & $203(5)$ & $2(3)$ & $59(3)$ & $-10(2)$ \\
\hline$C(18)$ & $60(2)$ & 85 (2) & $58(2)$ & $-6(1)$ & $22(1)$ & $6(1)$ \\
\hline$C(19)$ & $67(2)$ & $93(3)$ & $91(2)$ & $8(2)$ & $33(2)$ & $16(2)$ \\
\hline$c(20)$ & $63(2)$ & $116(3)$ & $94(2)$ & $15(2)$ & $32(2)$ & $17(2)$ \\
\hline$C(21)$ & $62(2)$ & $123(3)$ & $65(2)$ & $7(2)$ & $23(2)$ & $-10(2)$ \\
\hline$C(22)$ & $78(2)$ & $184(6)$ & $106(3)$ & $39(4)$ & $8(2)$ & $12(3)$ \\
\hline$C(9 A)$ & $56(2)$ & $53(2)$ & $64(2)$ & $-4(1)$ & $23(1)$ & $2(1)$ \\
\hline
\end{tabular}


Table 5. Hydrogen coordinates $\left(x 10^{\wedge} 4\right)$ and isotropic

displacement parameters $\left(A^{\wedge} 2 \times 10^{\wedge} 3\right)$ for cd24435.

\begin{tabular}{|c|c|c|c|c|}
\hline & $\mathrm{x}$ & y & $z$ & $\mathrm{U}(\mathrm{eq})$ \\
\hline $\mathrm{H}(1)$ & 448 & 2101 & 3247 & 78 \\
\hline $\mathrm{H}(2)$ & 2497 & 1055 & 3901 & 81 \\
\hline $\mathrm{H}(5 \mathrm{~A})$ & 1691 & 5077 & 721 & 74 \\
\hline$H(5 B)$ & 2982 & 5123 & 1398 & 74 \\
\hline$H(6 A)$ & 2476 & 7606 & 2376 & 82 \\
\hline$H(6 B)$ & 2480 & 8210 & 1297 & 82 \\
\hline $\mathrm{H}(7 \mathrm{~A})$ & 917 & 9540 & 1574 & 79 \\
\hline$H(7 B)$ & 496 & 8058 & 680 & 79 \\
\hline $\mathrm{H}(8)$ & 633 & 7200 & 2692 & 68 \\
\hline $\mathrm{H}\langle 9\rangle$ & -166 & 4904 & 930 & 63 \\
\hline $\mathrm{H}(10)$ & -1001 & 4476 & 2578 & 64 \\
\hline $\mathrm{H}(12)$ & -2563 & 7125 & 2706 & 69 \\
\hline $\mathrm{H}(15 \mathrm{~A})$ & -4806 & 7489 & 2065 & 1.28 \\
\hline$H(15 B)$ & -5375 & 6920 & 921 & 128 \\
\hline $\mathrm{H}(15 \mathrm{C})$ & -5011 & 9059 & 1216 & 128 \\
\hline $\mathrm{H}(16 \mathrm{~A})$ & -2131 & 3171 & 584 & 90 \\
\hline $\mathrm{H}(16 \mathrm{~B})$ & -1293 & 1835 & 1400 & 90 \\
\hline $\mathrm{H}(17 \mathrm{~A})$ & -2498 & 2068 & 2401 & 137 \\
\hline $\mathrm{H}(17 \mathrm{~B})$ & -3151 & 1170 & 1335 & 137 \\
\hline $\mathrm{H}(17 \mathrm{C})$ & -3348 & 3333 & 1553 & 137 \\
\hline $\mathrm{H}(18)$ & 3991 & 2276 & 2218 & 81 \\
\hline $\mathrm{H}(19 \mathrm{~A})$ & 4658 & 14 & 3399 & 98 \\
\hline $\mathrm{H}(19 \mathrm{~B})$ & 4437 & 1266 & 4263 & 98 \\
\hline $\mathrm{H}(20)$ & 6225 & 1669 & 3444 & 108 \\
\hline $\mathrm{H}(22 \mathrm{~A})$ & 7308 & 2594 & 5166 & 132 \\
\hline $\mathrm{H}(22 \mathrm{~B})$ & 6940 & 424 & 5025 & 132 \\
\hline $\mathrm{H}(22 \mathrm{C})$ & 6263 & 1882 & 5472 & 132 \\
\hline
\end{tabular}


Table 6. Torsion angles [deg] for cd24435.

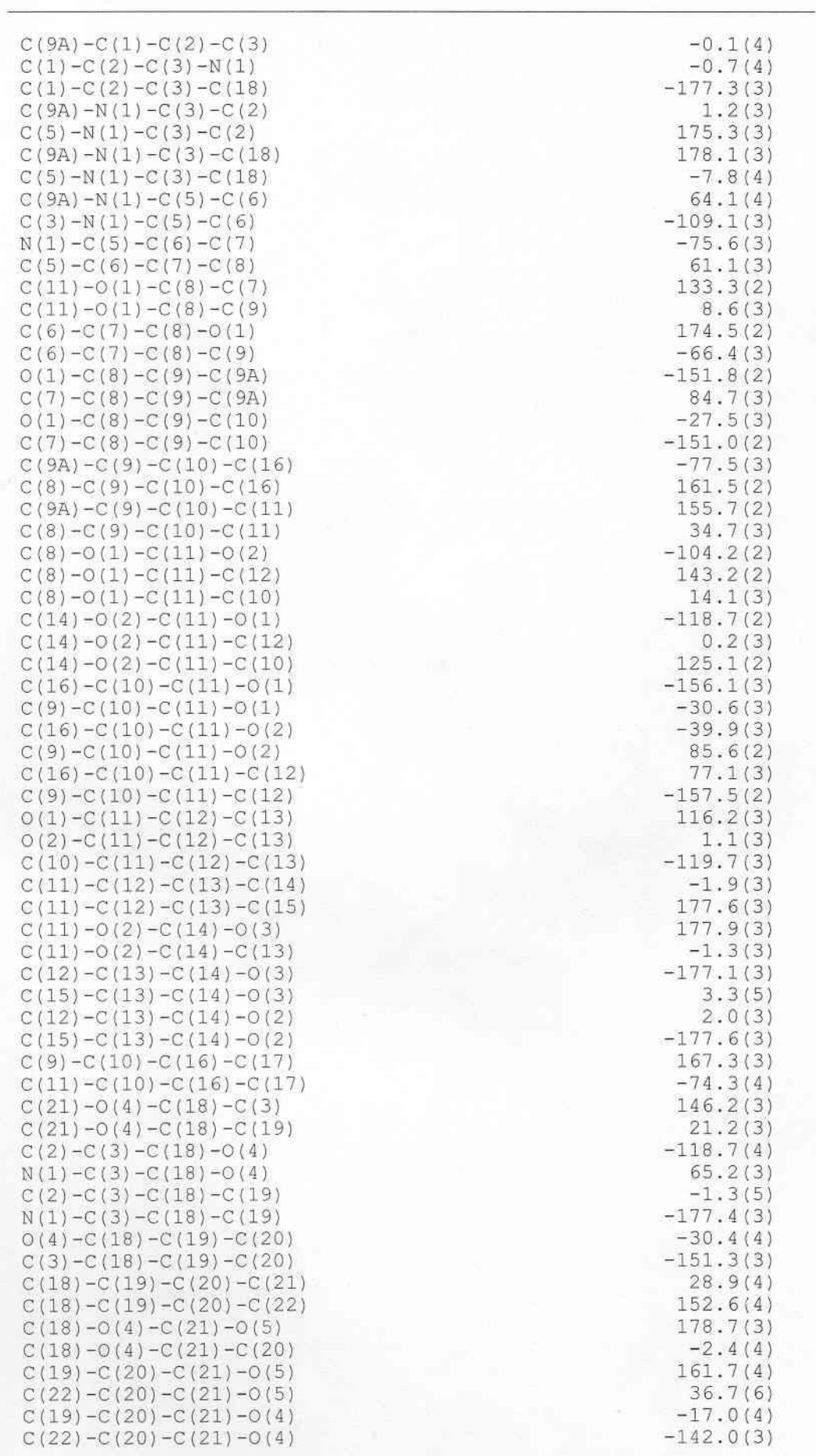




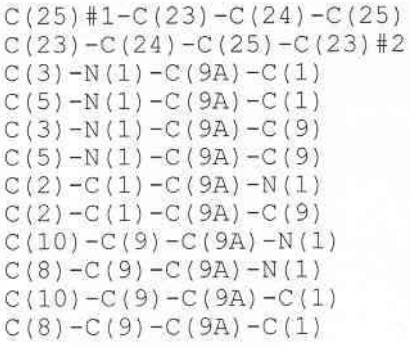

$-174.3(8)$

$175.8(9)$

$-1.3(3)$

$-175.6(3)$

$172.4(2)$

$-1.9(4)$

$0.9(3)$

$-172.0(3)$

$178.8(2)$

$-64.2(3)$

$-9.1(4)$

$107.9(3)$

Symmetry transformations used to generate equivalent atoms: $\# 1-x+2, y+1 / 2,-z+1 \quad \# 2-x+2, y-1 / 2,-z+1$ 
Table 7. Hydrogen bonds for cd24435 [A and deg.].

\begin{tabular}{lllll}
\hline$D-H \ldots A$ & $d(D-H)$ & $d(H \ldots A)$ & $d(D \ldots A)$ & $<(D H A)$
\end{tabular}

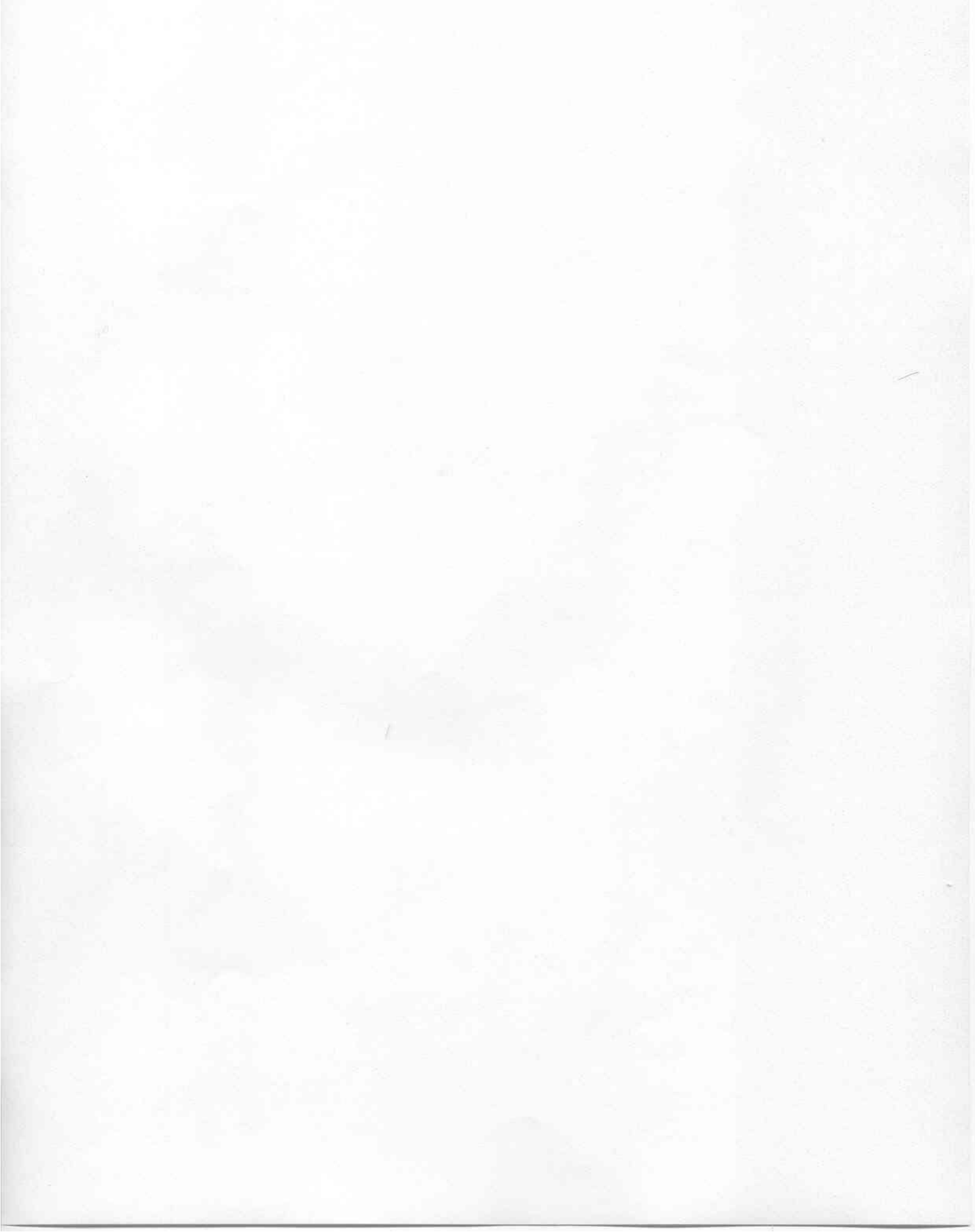




\section{X-ray diffraction data tables for bisdehydrostemoninine A (4)}

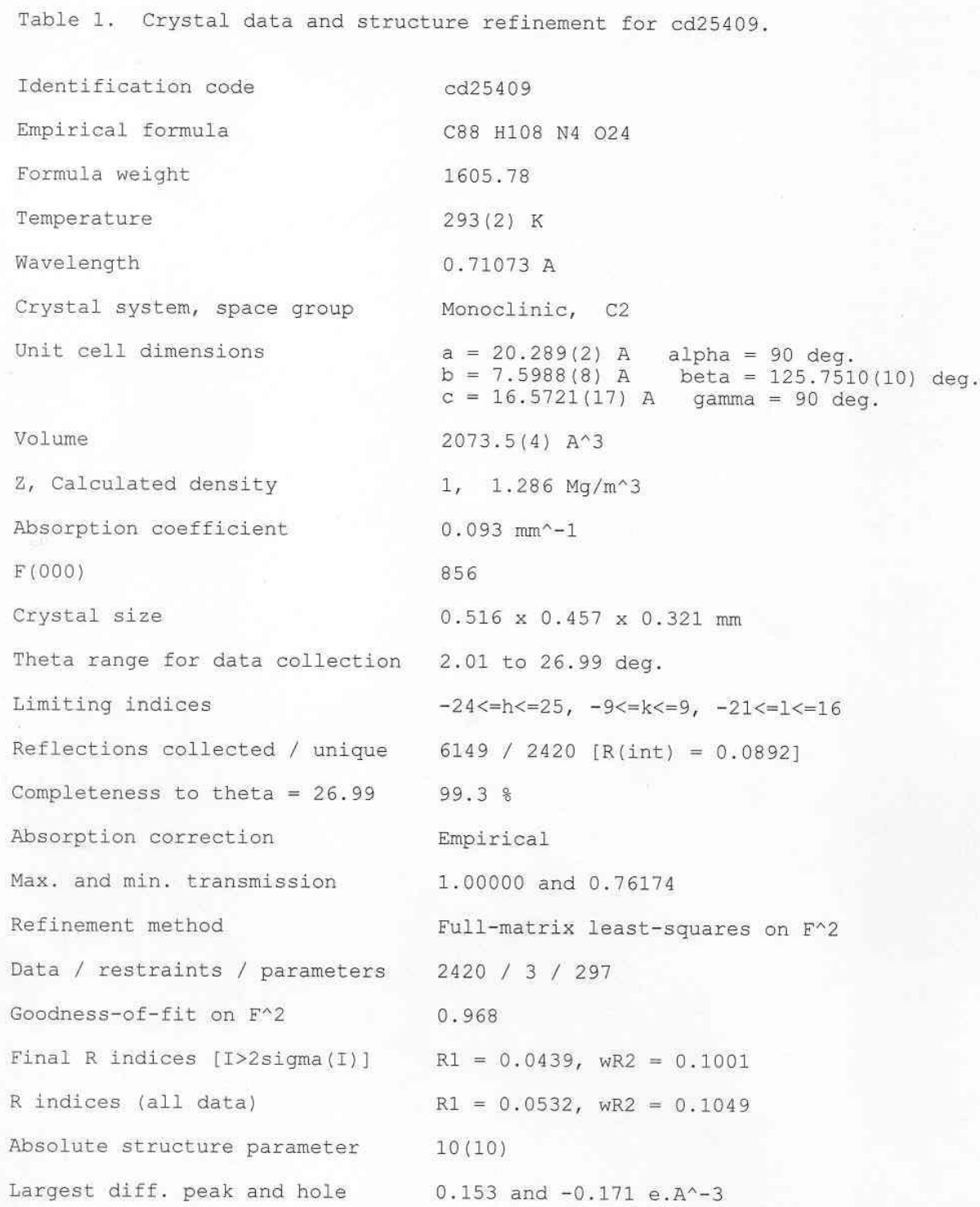


Table 2. Atomic coordinates $\left(\times 10^{\wedge} 4\right)$ and equivalent isotropic displacement parameters $\left(A^{\wedge} 2 \times 10^{\wedge} 3\right)$ for $c d 25409$.

$\mathrm{U}(\mathrm{eq})$ is defined as one third of the trace of the orthogonalized Uij tensor.

\begin{tabular}{|c|c|c|c|c|}
\hline & $x$ & y & $z$ & $\mathrm{U}(\mathrm{eq})$ \\
\hline$O(1)$ & $7517(1)$ & $9610(3)$ & $10753(1)$ & $62(1)$ \\
\hline $0(2)$ & $6948(1)$ & $11080(3)$ & $11426(2)$ & $63(1)$ \\
\hline $0(3)$ & $7217(2)$ & $13271(4)$ & $12469(3)$ & $113(1)$ \\
\hline$O(4)$ & $4401(2)$ & $5411(3)$ & $6327(2)$ & $88(1)$ \\
\hline$O(5)$ & $4994(1)$ & $1737(4)$ & $5997(1)$ & $75(1)$ \\
\hline $0(6)$ & $3940(1)$ & $1907(4)$ & $4439(1)$ & $82(1)$ \\
\hline$N(1)$ & $5380(1)$ & $6545(3)$ & $8408(1)$ & $45(1)$ \\
\hline$C(1)$ & $5768(2)$ & $4833(4)$ & $9693(2)$ & $53(1)$ \\
\hline$C(2)$ & $5273(2)$ & $3861(4)$ & $8837(2)$ & $53(1)$ \\
\hline$c(3)$ & $5030(2)$ & $4913(3)$ & $8033(2)$ & $48(1)$ \\
\hline$c(5)$ & $5270(2)$ & $8147(4)$ & $7850(2)$ & $50(1)$ \\
\hline$c(6)$ & $6036(2)$ & $8804(4)$ & $7997(2)$ & $55(1)$ \\
\hline$C(7)$ & $6630(2)$ & $9773(4)$ & $8966(2)$ & $56(1)$ \\
\hline$C(8)$ & $6928(2)$ & $8649(4)$ & $9867(2)$ & $48(1)$ \\
\hline$C(9)$ & $6277(1)$ & $8092(4)$ & $10006(2)$ & $44(1)$ \\
\hline$C(10)$ & $6765(2)$ & $7934(4)$ & $11136(2)$ & $45(1)$ \\
\hline$C(11)$ & $7350(2)$ & $9474(4)$ & $11460(2)$ & $48(1)$ \\
\hline$C(12)$ & $8130(2)$ & $9509(5)$ & $12476(2)$ & $57(1)$ \\
\hline$C(13)$ & $8175(2)$ & $10922(5)$ & $12967(2)$ & $65(1)$ \\
\hline$C(14)$ & $7418(2)$ & $11937(5)$ & $12303(3)$ & $69(1)$ \\
\hline$C(15)$ & $8839(3)$ & $11562(7)$ & $13973(3)$ & $105(2)$ \\
\hline$C(16)$ & $6264(2)$ & $7889(4)$ & $11554(2)$ & $54(1)$ \\
\hline$C(17)$ & $6747(2)$ & $7537(5)$ & $12648(2)$ & $63(1)$ \\
\hline$C(18)$ & $4515(2)$ & $4448(4)$ & $6981(2)$ & $53(1)$ \\
\hline$C(19)$ & $4105(2)$ & $2678(4)$ & $6720(2)$ & $55(1)$ \\
\hline$C(20)$ & $3681(2)$ & $2185(4)$ & $5631(2)$ & $53(1)$ \\
\hline$C(21)$ & $4275(2)$ & $1921(4)$ & $5390(2)$ & $50(1)$ \\
\hline$C(22)$ & $3159(2)$ & $521(5)$ & $5355(3)$ & $78(1)$ \\
\hline$C(9 A)$ & $5825(2)$ & $6490(4)$ & $9419(2)$ & $45(1)$ \\
\hline
\end{tabular}


Table 3. Bond lengths [A] and angles [deg] for cd25409.

\begin{tabular}{|c|c|}
\hline $\begin{array}{l}O(1)-C(11) \\
O(1)-C(8) \\
O(2)-C(14) \\
O(2)-C(11) \\
O(3)-C(14) \\
O(4)-C(18) \\
O(5)-C(21) \\
O(6)-C(21) \\
O(6)-H(6) \\
N(1)-C(9 A) \\
N(1)-C(3) \\
N(1)-C(5) \\
C(1)-C(9 A) \\
C(1)-C(2) \\
C(1)-H(1) \\
C(2)-C(3) \\
C(2)-H(2) \\
C(3)-C(18) \\
C(5)-C(6) \\
C(5)-H(5 A) \\
C(5)-H(5 B) \\
C(6)-C(7) \\
C(6)-H(6 A) \\
C(6)-H(6 B) \\
C(7)-C(8) \\
C(7)-H(7 A) \\
C(7)-H(7 B) \\
C(8)-C(9) \\
C(8)-H(8) \\
C(9)-C(9 A) \\
C(9)-C(10) \\
C(9)-H(9) \\
C(10)-C(11) \\
C(10)-C(16) \\
C(10)-H(10) \\
C(11)-C(12) \\
C(12)-C(13) \\
C(12)-H(12) \\
C(13)-C(14) \\
C(13)-C(15) \\
C(15)-H(15 A) \\
C(15)-H(15 B) \\
C(15)-H(15 C) \\
C(16)-C(17) \\
C(16)-H(16 A) \\
C(16)-H(16 B) \\
C(17)-H(17 A) \\
C(17)-H(17 B) \\
C(17)-H(17 C) \\
C(18)-C(19) \\
C(19)-C(20) \\
C(19)-H(19 A) \\
C(19)-H(19 B) \\
C(20)-C(21) \\
C(20)-C(22) \\
C(20)-H(20) \\
C(22)-H(22 A) \\
C(22)-H(22 B) \\
C(22)-H(22 C)\end{array}$ & $\begin{array}{l}1.400(3) \\
1.438(3) \\
1.351(4) \\
1.451(4) \\
1.183(5) \\
1.213(3) \\
1.202(3) \\
1.301(3) \\
1.01(6) \\
1.363(3) \\
1.383(4) \\
1.465(3) \\
1.366(4) \\
1.379(4) \\
0.936(18) \\
1.374(4) \\
0.902(18) \\
1.459(4) \\
1.512(4) \\
0.9700 \\
0.9700 \\
1.520(4) \\
0.9700 \\
0.9700 \\
1.507(4) \\
0.9700 \\
0.9700 \\
1.526(3) \\
0.94(3) \\
1.492(4) \\
1.528(3) \\
0.98(3) \\
1.524(4) \\
1.529(3) \\
0.95(3) \\
1.492(4) \\
1.318(5) \\
0.90(3) \\
1.479(5) \\
1.485(5) \\
0.9600 \\
0.9600 \\
0.9600 \\
1.498(4) \\
0.9700 \\
0.9700 \\
0.9600 \\
0.9600 \\
0.9600 \\
1.506(4) \\
1.524(4) \\
0.9700 \\
0.9700 \\
1.488(4) \\
1.538(4) \\
0.95(4) \\
0.9600 \\
0.9600 \\
0.9600 \\
\end{array}$ \\
\hline $\begin{array}{l}C(11)-O(1)-C(8) \\
C(14)-O(2)-C(11) \\
C(21)-O(6)-H(6) \\
C(9 A)-N(1)-C(3) \\
C(9 A)-N(1)-C(5) \\
C(3)-N(1)-C(5) \\
C(9 A)-C(1)-C(2) \\
C(9 A)-C(1)-H(1)\end{array}$ & $\begin{array}{l}110.7(2) \\
109.6(2) \\
113(3) \\
108.8(2) \\
123.3(2) \\
127.8(2) \\
107.7(2) \\
125(2)\end{array}$ \\
\hline
\end{tabular}




\begin{tabular}{|c|c|}
\hline $\begin{array}{l}C(2)-C(1)-H(1) \\
C(3)-C(2)-C(1) \\
C(3)-C(2)-H(2) \\
C(1)-C(2)-H(2) \\
C(2)-C(3)-N(1) \\
C(2)-C(3)-C(18) \\
N(1)-C(3)-C(18) \\
N(1)-C(5)-C(6) \\
N(1)-C(5)-H(5 A) \\
C(6)-C(5)-H(5 A) \\
N(1)-C(5)-H(5 B) \\
C(6)-C(5)-H(5 B) \\
H(5 A)-C(5)-H(5 B) \\
C(5)-C(6)-C(7) \\
C(5)-C(6)-H(6 A) \\
C(7)-C(6)-H(6 A) \\
C(5)-C(6)-H(6 B) \\
C(7)-C(6)-H(6 B) \\
H(6 A)-C(6)-H(6 B) \\
C(8)-C(7)-C(6) \\
C(8)-C(7)-H(7 A) \\
C(6)-C(7)-H(7 A) \\
C(8)-C(7)-H(7 B) \\
C(6)-C(7)-H(7 B) \\
H(7 A)-C(7)-H(7 B) \\
O(1)-C(8)-C(7) \\
O(1)-C(8)-C(9) \\
C(7)-C(8)-C(9) \\
O(1)-C(8)-H(8) \\
C(7)-C(8)-H(8) \\
C(9)-C(8)-H(8) \\
C(9 A)-C(9)-C(8) \\
C(9 A)-C(9)-C(10) \\
C(8)-C(9)-C(10) \\
C(9 A)-C(9)-H(9) \\
C(8)-C(9)-H(9) \\
C(10)-C(9)-H(9) \\
C(11)-C(10)-C(16) \\
C(11)-C(10)-C(9) \\
C(16)-C(10)-C(9) \\
C(11)-C(10)-H(10) \\
C(16)-C(10)-H(10) \\
C(9)-C(10)-H(10) \\
O(1)-C(11)-O(2) \\
O(1)-C(11)-C(12) \\
O(2)-C(11)-C(12) \\
O(1)-C(11)-C(10) \\
O(2)-C(11)-C(10) \\
C(12)-C(11)-C(10) \\
C(13)-C(12)-C(11) \\
C(13)-C(12)-H(12) \\
C(11)-C(12)-H(12) \\
C(12)-C(13)-C(14) \\
C(12)-C(13)-C(15) \\
C(14)-C(13)-C(15) \\
O(3)-C(14)-O(2) \\
O(3)-C(14)-C(13) \\
O(2)-C(14)-C(13) \\
C(13)-C(15)-H(15 A) \\
C(13)-C(15)-H(15 B) \\
H(15 A)-C(15)-H(15 B) \\
C(13)-C(15)-H(15 C) \\
H(15 A)-C(15)-H(15 C) \\
H(15 B)-C(15)-H(15 C) \\
C(17)-C(16)-C(10) \\
C(17)-C C(16)-H(16 A) \\
C(10)-C(16)-H(16 A) \\
C(17)-C(16)-H(16 B) \\
C(10)-C(16)-H(16 B) \\
H(16 A)-C(16)-H(16 B) \\
C(16)-C(17)-H(17 A) \\
C(16)-C(17)-H(17 B) \\
C(1)-H)\end{array}$ & 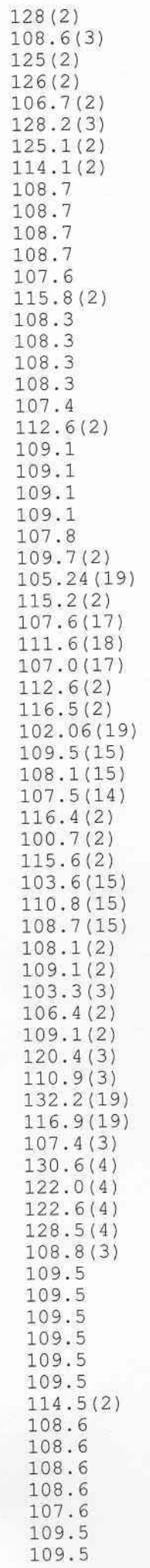 \\
\hline
\end{tabular}




\begin{tabular}{|c|c|}
\hline$H(17 A)-C(17)-H(17 B)$ & 109.5 \\
\hline$C(16)-C(17)-H(17 C)$ & 109.5 \\
\hline $\mathrm{H}(17 \mathrm{~A})-\mathrm{C}(17)-\mathrm{H}(17 \mathrm{C})$ & 109.5 \\
\hline $\mathrm{H}(17 \mathrm{~B})-\mathrm{C}(17)-\mathrm{H}(17 \mathrm{C})$ & 109.5 \\
\hline$O(4)-C(18)-C(3)$ & $123.5(3)$ \\
\hline$O(4)-C(18)-C(19)$ & $119.8(3)$ \\
\hline$C(3)-C(18)-C(19)$ & $116.7(2)$ \\
\hline$C(18)-C(19)-C(20)$ & $113.2(2)$ \\
\hline$C(18)-C(19)-H(19 A)$ & 108.9 \\
\hline$C(20)-C(19)-H(19 A)$ & 108.9 \\
\hline$C(18)-C(19)-H(19 B)$ & 108.9 \\
\hline$C(20)-C(19)-H(19 B)$ & 108.9 \\
\hline $\mathrm{H}(19 \mathrm{~A})-\mathrm{C}(19)-\mathrm{H}(19 \mathrm{~B})$ & 107.7 \\
\hline$C(21)-C(20)-C(19)$ & $111.5(2)$ \\
\hline$C(21)-C(20)-C(22)$ & $109.5(3)$ \\
\hline$C(19)-C(20)-C(22)$ & $111.6(3)$ \\
\hline$C(21)-C(20)-H(20)$ & $107.1(19)$ \\
\hline$C(19)-C(20)-H(20)$ & $106(2)$ \\
\hline$C(22)-C(20)-H(20)$ & $111(2)$ \\
\hline$O(5)-C(21)-O(6)$ & $122.1(3)$ \\
\hline$O(5)-C(21)-C(20)$ & $124.6(2)$ \\
\hline$O(6)-C(21)-C(20)$ & $113.3(2)$ \\
\hline$C(20)-C(22)-H(22 A)$ & 109.5 \\
\hline$C(20)-C(22)-H(22 B)$ & 109.5 \\
\hline$H(22 A)-C(22)-H(22 B)$ & 109.5 \\
\hline $\mathrm{C}(20)-\mathrm{C}(22)-\mathrm{H}(22 \mathrm{C})$ & 109.5 \\
\hline $\mathrm{H}(22 \mathrm{~A})-\mathrm{C}(22)-\mathrm{H}(22 \mathrm{C})$ & 109.5 \\
\hline$H(22 B)-C(22)-H(22 C)$ & 109.5 \\
\hline$N(1)-C(9 A)-C(1)$ & $108.2(2)$ \\
\hline$N(1)-C(9 A)-C(9)$ & $119.3(2)$ \\
\hline$C(1)-C(9 A)-C(9)$ & $132.4(2)$ \\
\hline
\end{tabular}

Symmetry transformations used to generate equivalent atoms: 
Table 4. Anisotropic displacement parameters $\left(A^{\wedge} 2 \times 10^{\wedge} 3\right.$ ) for $\mathrm{cd}_{2} 5409$. The anisotropic displacement factor exponent takes the form:

$-2 \mathrm{pi}^{\wedge} 2\left[\mathrm{~h}^{\wedge} 2 \mathrm{a}^{\star \wedge} 2 \mathrm{U} 11+\ldots+2 \mathrm{~h} k \mathrm{a}^{\star} \mathrm{b}^{\star} \mathrm{U} 12\right]$

\begin{tabular}{|c|c|c|c|c|c|c|}
\hline & U11 & $\mathrm{U} 22$ & U33 & U23 & U13 & U12 \\
\hline$O(1)$ & $57(1)$ & $90(1)$ & $47(1)$ & $-6(1)$ & $35(1)$ & $-17(1)$ \\
\hline$O(2)$ & $64(1)$ & $61(1)$ & $60(1)$ & $O(1)$ & $34(1)$ & $3(1)$ \\
\hline $0(3)$ & $140(3)$ & $81(2)$ & $140(3)$ & $-35(2)$ & $95(2)$ & $-6(2)$ \\
\hline$O(4)$ & $139(2)$ & $70(2)$ & $47(1)$ & $-3(1)$ & $50(1)$ & $-27(2)$ \\
\hline$O(5)$ & $61(1)$ & $123(2)$ & $42(1)$ & $18(1)$ & $30(1)$ & $22(1)$ \\
\hline $0(6)$ & 61 (1) & $140(2)$ & $39(1)$ & $-5(1)$ & $27(1)$ & $-8(2)$ \\
\hline$N(1)$ & $50(1)$ & $48(1)$ & $38(1)$ & $6(1)$ & $27(1)$ & $4(1)$ \\
\hline$C(1)$ & $62(2)$ & $57(2)$ & $42(2)$ & $10(1)$ & $32(1)$ & $6(1)$ \\
\hline$C(2)$ & $67(2)$ & $48(2)$ & $51(2)$ & $4(1)$ & $38(1)$ & $2(1)$ \\
\hline$C(3)$ & $54(1)$ & $49(2)$ & $44(1)$ & $5(1)$ & $31(1)$ & $4(1)$ \\
\hline$C(5)$ & $57(1)$ & $47(1)$ & 41 (1) & $10(1)$ & $26(1)$ & $8(1)$ \\
\hline$c(6)$ & $71(2)$ & $57(2)$ & $44(1)$ & $10(1)$ & $37(1)$ & $4(1)$ \\
\hline$C(7)$ & $61(2)$ & $65(2)$ & $53(2)$ & $4(1)$ & $39(1)$ & $-2(1)$ \\
\hline$c(8)$ & $49(1)$ & $60(2)$ & $42(1)$ & $5(1)$ & $30(1)$ & $5(1)$ \\
\hline$C(9)$ & $44(1)$ & 51 (1) & 41 (1) & $6(1)$ & $27(1)$ & $8(1)$ \\
\hline$c(10)$ & $45(1)$ & $55(2)$ & $43(1)$ & $2(1)$ & $30(1)$ & $5(1)$ \\
\hline$C(11)$ & $47(1)$ & $60(2)$ & $43(1)$ & $3(1)$ & $29(1)$ & $4(1)$ \\
\hline$C(12)$ & $51(2)$ & $71(2)$ & $50(2)$ & $3(2)$ & $29(1)$ & $-6(2)$ \\
\hline$C(13)$ & $72(2)$ & $77(2)$ & $48(2)$ & $-5(2)$ & $36(2)$ & $-22(2)$ \\
\hline$C(14)$ & $89(2)$ & $62(2)$ & $78(2)$ & $-8(2)$ & $61(2)$ & $-10(2)$ \\
\hline$C(15)$ & $124(3)$ & $120(3)$ & $53(2)$ & $-19(2)$ & $42(2)$ & $-45(3)$ \\
\hline$C(16)$ & $52(1)$ & $71(2)$ & $52(2)$ & $-3(1)$ & $38(1)$ & $-3(1)$ \\
\hline$C(17)$ & $71(2)$ & $83(2)$ & $53(2)$ & $-4(2)$ & $45(2)$ & $-7(2)$ \\
\hline$C(18)$ & $65(2)$ & $55(2)$ & $46(1)$ & $1(1)$ & $37(1)$ & $0(1)$ \\
\hline$C(19)$ & $64(2)$ & $62(2)$ & $48(2)$ & $-1(1)$ & $38(1)$ & $-3(1)$ \\
\hline$C(20)$ & $53(1)$ & $58(2)$ & $43(1)$ & $2(1)$ & $26(1)$ & $-2(1)$ \\
\hline$C(21)$ & $55(2)$ & $50(2)$ & $37(1)$ & $1(1)$ & $23(1)$ & $-5(1)$ \\
\hline$C(22)$ & $80(2)$ & $91(3)$ & $62(2)$ & $-11(2)$ & $41(2)$ & $-34(2)$ \\
\hline$C(9 A)$ & $45(1)$ & $53(2)$ & $40(1)$ & $5(1)$ & 27 (1) & $8(1)$ \\
\hline
\end{tabular}


Table 5. Hydrogen coordinates $\left(x 10^{\wedge} 4\right)$ and isotropic displacement parameters $\left(A^{\wedge} 2 \times 10^{\wedge} 3\right)$ for cd25409.

\begin{tabular}{|c|c|c|c|c|}
\hline & $x$ & $y$ & z & $\mathrm{U}(\mathrm{eq})$ \\
\hline $\mathrm{H}(5 \mathrm{~A})$ & 5060 & 9067 & 8049 & 60 \\
\hline $\mathrm{H}(5 \mathrm{~B})$ & 4865 & 7923 & 7148 & 60 \\
\hline $\mathrm{H}(6 \mathrm{~A})$ & 5884 & 9584 & 7450 & 66 \\
\hline $\mathrm{H}(6 \mathrm{~B})$ & 6315 & 7805 & 7960 & 66 \\
\hline $\mathrm{H}(7 \mathrm{~A})$ & 7092 & 10156 & 8978 & 67 \\
\hline $\mathrm{H}(7 \mathrm{~B})$ & 6367 & 10813 & 8994 & 67 \\
\hline $\mathrm{H}(15 \mathrm{~A})$ & 9354 & 11225 & 14117 & 127 \\
\hline $\mathrm{H}(15 \mathrm{~B})$ & 8780 & 11055 & 14458 & 127 \\
\hline $\mathrm{H}(15 \mathrm{C})$ & 8812 & 12821 & 13993 & 127 \\
\hline $\mathrm{H}(16 \mathrm{~A})$ & 5988 & 9010 & 11418 & 65 \\
\hline $\mathrm{H}(16 \mathrm{~B})$ & 5851 & 6986 & 11207 & 65 \\
\hline $\mathrm{H}(17 \mathrm{~A})$ & 7096 & 8522 & 13006 & 95 \\
\hline $\mathrm{H}(17 \mathrm{~B})$ & 7071 & 6499 & 12804 & 95 \\
\hline $\mathrm{H}(17 \mathrm{C})$ & 6385 & 7365 & 12837 & 95 \\
\hline H (19A) & 3708 & 2684 & 6869 & 66 \\
\hline $\mathrm{H}(19 \mathrm{~B})$ & 4507 & 1787 & 7133 & 66 \\
\hline$H(22 A)$ & 3504 & -471 & 5707 & 118 \\
\hline $\mathrm{H}(22 \mathrm{~B})$ & 2857 & 319 & 4652 & 118 \\
\hline $\mathrm{H}(22 \mathrm{C})$ & 2789 & 676 & 5533 & 118 \\
\hline $\mathrm{H}(1)$ & $6014(16)$ & $4470(40)$ & $10349(15)$ & $62(8)$ \\
\hline $\mathrm{H}(2)$ & $5117(17)$ & $2730(30)$ & $8800(20)$ & $56(8)$ \\
\hline$H(6)$ & $4300(30)$ & $1360(90)$ & $4260(40)$ & $110(20)$ \\
\hline $\mathrm{H}(8)$ & $7179(15)$ & $7620(40)$ & $9860(20)$ & $49(8)$ \\
\hline$H(9)$ & $5898(15)$ & $9070(30)$ & $9801(18)$ & $37(6)$ \\
\hline $\mathrm{H}(10)$ & $7107(14)$ & $6930(40)$ & $11344(19)$ & $41(7)$ \\
\hline $\mathrm{H}(12)$ & $8475(18)$ & $8610(40)$ & $12640(20)$ & $55(8)$ \\
\hline $\mathrm{H}(20)$ & 3359 (19) & $3170(50)$ & $5260(30)$ & $66(9)$ \\
\hline
\end{tabular}


Table 6. Torsion angles [deg] for cd25409.

\begin{tabular}{|c|c|}
\hline $\begin{array}{l}C(9 A)-C(1)-C(2)-C(3) \\
C(1)-C(2)-C(3)-N(1) \\
C(1)-C(2)-C(3)-C(18) \\
C(9 A)-N(1)-C(3)-C(2) \\
C(5)-N(1)-C(3)-C(2) \\
C(9 A)-N(1)-C(3)-C(18) \\
C(5)-N(1)-C(3)-C(18) \\
C(9 A)-N(1)-C(5)-C(6) \\
C(3)-N(1)-C(5)-C(6) \\
N(1)-C(5)-C(6)-C(7) \\
C(5)-C(6)-C(7)-C(8) \\
C(11)-O(1)-C(8)-C(7) \\
C(11)-O(1)-C(8)-C(9) \\
C(6)-C(7)-C(8)-O(1) \\
C(6)-C(7)-C(8)-C(9) \\
O(1)-C(8)-C(9)-C(9 A) \\
C(7)-C(8)-C(9)-C(9 A) \\
O(1)-C(8)-C(9)-C(10) \\
C(7)-C(8)-C(9)-C(10) \\
C(9 A)-C(9)-C(10)-C(11) \\
C(8)-C(9)-C(10)-C(11) \\
C(9 A)-C(9)-C(10)-C(16) \\
C(8)-C(9)-C(10)-C(16) \\
C(8)-O(1)-C(11)-O(2) \\
C(8)-O(1)-C(11)-C(12) \\
C(8)-O(1)-C(11)-C(10) \\
C(14)-O(2)-C(11)-O(1) \\
C(14)-O(2)-C(11)-C(12) \\
C(14)-O(2)-C(11)-C(10) \\
C(16)-C(10)-C(11)-O(1) \\
C(9)-C(10)-C(11)-O(1) \\
C(16)-C(10)-C(11)-O(2) \\
C(9)-C(10)-C(11)-O(2) \\
C(16)-C(10)-C(11)-C(12) \\
C(9)-C(10)-C(11)-C(12) \\
O(1)-C(11)-C(12)-C(13) \\
O(2)-C(11)-C(12)-C(13) \\
C(10)-C(11)-C(12)-C(13) \\
C(11)-C(12)-C(13)-C(14) \\
C(11)-C(12)-C(13)-C(15) \\
C(11)-O(2)-C(14)-O(3) \\
C C(11)-O(2)-C(14)-C(13) \\
C(12)-C(13)-C(14)-O(3) \\
C(15)-C(13)-C(14)-O(3) \\
C C(12)-C(13)-C(14)-O(2) \\
C(15)-C(13)-C(14)-O(2) \\
C(11)-C(10)-C(16)-C(17) \\
C(9)-C(10)-C(16)-C(17) \\
C(2)-C(3)-C(18)-O(4) \\
N(1)-C(3)-C(18)-O(4) \\
C(2)-C(3)-C(18)-C(19) \\
N(1)-C(3)-C(18)-C(19) \\
O(4)-C(18)-C(19)-C(20) \\
C(3)-C(18)-C(19)-C(20) \\
C(18)-C(19)-C(20)-C(21) \\
C(18)-C(19)-C(20)-C(22) \\
C(19)-C(20)-C(21)-O(5) \\
C(22)-C(20)-C(21)-O(5) \\
C(19)-C(20)-C(21)-O(6) \\
C(22)-C(20)-C(21)-O(6) \\
C(3)-N(1)-C(9 A)-C(1) \\
C(5)-N(1)-C(9 A)-C(1) \\
C(3)-N(1)-C(9 A)-C(9) \\
C(5)-N(1)-C(9 A)-C(9) \\
C(2)-C C(1)-C(9 A)-N(1) \\
C(2)-C C(1)-C(9 A)-C(9) \\
C(8)-C(9)-C(9 A)-N(1) \\
C(10)-C(9)-C(9 A)-N(1)\end{array}$ & $\begin{array}{r}0.6(3) \\
-0.2(3) \\
178.8(3) \\
-0.3(3) \\
-176.2(2) \\
-179.4(2) \\
4.7(4) \\
67.5(3) \\
-117.1(3) \\
-77.6(3) \\
60.0(3) \\
132.9(2) \\
8.4(3) \\
177.0(2) \\
-64.5(3) \\
-154.9(2) \\
84.1(3) \\
-29.2(3) \\
-150.2(2) \\
160.5(2) \\
37.4(3) \\
-73.2(3) \\
163.7(3) \\
-100.7(3) \\
147.6(3) \\
16.3(3) \\
-116.2(2) \\
-0.7(3) \\
128.5(2) \\
-159.5(2) \\
-33.8(3) \\
-43.2(3) \\
82.6(2) \\
75.9(3) \\
-158.4(2) \\
115.3(3) \\
0.4(3) \\
-121.5(3) \\
0.0(3) \\
-177.2(3) \\
-178.6(3) \\
0.7(3) \\
178.8(3) \\
-3.8(6) \\
-0.4(3) \\
177.0(3) \\
-68.5(4) \\
173.6(3) \\
-172.0(3) \\
6.9(4) \\
8.9(4) \\
-172.2(2) \\
9.2(4) \\
-171.6(2) \\
66.2(3) \\
-171.0(3) \\
15.1(5) \\
-109.0(4) \\
-164.8(3) \\
71.1(4) \\
0.6(3) \\
176.8(2) \\
179.1(2) \\
-4.7(3) \\
-0.8(3) \\
-178.9(3) \\
-62.4(3) \\
-179.8(2)\end{array}$ \\
\hline
\end{tabular}


$C(8)-C(9)-C(9 A)-C(1)$

$115.6(3)$

$C(10)-C(9)-C(9 A)-C(1)$

$-1.8(4)$

Symmetry transformations used to generate equivalent atoms: 
Table 7. Hydrogen bonds for cd25409 [A and deg.].

\begin{tabular}{lllll}
\hline$D-H \ldots A$ & $d(D-H)$ & $d(H \ldots A)$ & $d(D \ldots A)$ & $<(D H A)$ \\
$O(6)-H(6) \ldots O(5) \# 1$ & $1.01(6)$ & $1.75(5)$ & $2.653(3)$ & $146(6)$ \\
\hline
\end{tabular}

Symmetry transformations used to generate equivalent atoms: $\# 1-x+1, y,-z+1$ 